\title{
Different Estimation Procedures for the Parameters of the Extended Exponential Geometric Distribution for Medical Data
}

\author{
Francisco Louzada, ${ }^{1}$ Pedro L. Ramos, ${ }^{1}$ and Gleici S. C. Perdoná ${ }^{2}$ \\ ${ }^{1}$ Statistics Department, Institute of Mathematical and Computer Sciences (ICMC), São Paulo University (USP), \\ 13560-970 São Carlos, SP, Brazil \\ ${ }^{2}$ Department of Social Medicine, Ribeirão Preto School of Medicine (FMRP), São Paulo University (USP), \\ 14049-900 Ribeirão Preto, SP, Brazil \\ Correspondence should be addressed to Francisco Louzada; louzada@icmc.usp.br
}

Received 19 May 2016; Accepted 3 July 2016

Academic Editor: Ezequiel López-Rubio

Copyright ( $) 2016$ Francisco Louzada et al. This is an open access article distributed under the Creative Commons Attribution License, which permits unrestricted use, distribution, and reproduction in any medium, provided the original work is properly cited.

\begin{abstract}
We have considered different estimation procedures for the unknown parameters of the extended exponential geometric distribution. We introduce different types of estimators such as the maximum likelihood, method of moments, modified moments, $L$-moments, ordinary and weighted least squares, percentile, maximum product of spacings, and minimum distance estimators. The different estimators are compared by using extensive numerical simulations. We discovered that the maximum product of spacings estimator has the smallest mean square errors and mean relative estimates, nearest to one, for both parameters, proving to be the most efficient method compared to other methods. Combining these results with the good properties of the method such as consistency, asymptotic efficiency, normality, and invariance we conclude that the maximum product of spacings estimator is the best one for estimating the parameters of the extended exponential geometric distribution in comparison with its competitors. For the sake of illustration, we apply our proposed methodology in two important data sets, demonstrating that the EEG distribution is a simple alternative to be used for lifetime data.
\end{abstract}

\section{Introduction}

Many researches are interested in search distributions which can be used to describe real data sets. Generalizations of the standard exponential distribution have been introduced in the literature for this purpose, such as Gamma, Weibull, and Generalized Exponential distribution [1]. Another useful generalization is known as extended exponential geometric distribution. Initially, the development of such distribution was made by Adamidis and Loukas [2] proposing exponential geometric distribution with two parameters, in which the hazard function could be decreasing. In a further paper, Adamidis et al. [3] explored extended exponential geometric (EEG) distribution. Let $X$ be a random variable representing a lifetime data, with extended exponential geometric (EEG) distribution; its probability density function (PDF) is given by

$$
f(x \mid \gamma, \lambda)=\frac{\lambda \gamma e^{-\lambda x}}{\left(1-(1-\gamma) e^{-\lambda x}\right)^{2}},
$$

for all $x>0, \gamma>0$, and $\lambda>0$. One of its peculiarities is that its hazard function can be increasing or decreasing, depending on the values of its parameters, giving great flexibility of fit for real applications.

This model arises naturally in competing risks scenarios. Let $X=\min \left(T_{1}, T_{2}, \ldots, T_{M}\right)$, where $M$ is a random variable with geometrical distribution and $T_{i}$ are independent of $M$ and are assumed to be independent and identically distributed according to exponential distribution; then the random variable $X$ has EEG distribution with $0<\gamma<1$, also known as exponential geometric (EG) distribution [2]. Considering the same assumptions and $X=\max \left(T_{1}, T_{2}, \ldots, T_{M}\right)$, the random variable $X$ has EEG distribution with $\gamma>1$, also known as Complementary Exponential Geometric distribution [4]. Due to its importance, some generalizations of the EEG distribution have been proposed, such as the Beta exponential geometric distribution [5], Exponentiated Exponential-Geometric distribution 
[6], Complementary Exponentiated Exponential Geometric distribution [7], and Generalized Exponential Geometric distribution [8].

Despite the fact that EEG distribution has good flexibility, a few estimation procedures have been proposed in the literature. Adamidis et al. [3] derived the maximum likelihood estimators (MLE) for the unknown parameters of the EEG distribution. Ramos et al. [9] developed a Bayesian analysis under noninformative priors. However, considering the frequentist approach, it is well known that, usually, for small samples, the MLE does not perform well. In this paper, we proposed nine new estimators for the parameters of the EEG distribution, which are given considering the following estimation procedures: the method of moments, modified moments, ordinary least squares, weighted least squares, $L$-moments, percentile, maximum product of spacings, Cramer-von Mises type minimum distance, and Anderson-Darling estimator.

The main aim of this paper is twofold. First, it aims to develop a guideline for choosing the most efficient estimators among ten different estimation procedures for the EEG distribution, which would be of interest to applied statisticians. Second, it aims to demonstrate that the EEG distribution is a simple alternative to be used in applications in medicine.

The originality of this study comes from the fact that, for the EEG distribution and considering the frequentist approach, only the MLE has been presented in the literature. The performances of the different estimation methods are compared using extensive numerical simulations. Additionally, these results are analogous for the exponential geometric distribution and the Complementary Exponential Geometric distribution. Related studies for other distributions can be found in Gupta and Kundu [10], Mazucheli et al. [11], Teimouri et al. [12], and Dey et al. [13].

The paper is organized as follows. In Section 2, we discuss some properties of the EEG distribution. In Section 3, we present ten estimation procedures for the parameters of our proposed model. In Section 4, a simulation study is presented in order to identify the most efficient estimators. In Section 5, we apply our proposed methodology in two real data sets. Some final comments are presented in Section 6.

\section{Extended Exponential Geometric Distribution}

Let $X$ be a random variable with density function (1); the distribution function is given by

$$
F(x \mid \gamma, \lambda)=\frac{1-e^{-\lambda x}}{1-(1-\gamma) e^{-\lambda x}} .
$$

The survival and hazard functions of $\operatorname{EEG}(\gamma, \lambda)$ distribution is given, respectively, by

$$
\begin{aligned}
& S(x \mid \gamma, \lambda)=\frac{\gamma e^{-\lambda x}}{1-(1-\gamma) e^{-\lambda x}}, \\
& h(x \mid \gamma, \lambda)=\frac{\lambda}{1-(1-\gamma) e^{-\lambda x}} .
\end{aligned}
$$

The hazard function (3) is decreasing for $0<\gamma<1$, is constant for $\gamma=1$, and is monotonically increasing when $\gamma>1$. Figure 1 presents different forms for the density and hazard functions for the EEG distribution considering different values of $\gamma$ and $\lambda$.

For the random variable $X$ with EEG distribution, the moment generating function [14] is given by

$$
M_{X}(t)=1+\frac{t \gamma}{\lambda} \Phi\left(1-\gamma, 1,1-\frac{t}{\lambda}\right)
$$

for $t<\lambda$, where $\Phi(z, s, a)=\Gamma(s)^{-1} \int_{0}^{\infty} t^{s-1} e^{-a t}\left(1-z e^{-t}\right)^{-1} d t$, for $a, s>0$, and $z<1$ is known as Lerch transcendental function [15]. Note that the Laplace transform of the EEG distribution can be easily obtained from the relation $\operatorname{LT}_{X}(t)=$ $M_{X}(-t)=E\left(e^{-t X}\right)$. The raw moments of the EEG distribution are

$$
E\left(X^{r} \mid \gamma, \lambda\right)=r ! \gamma \lambda^{-r} \Phi(1-\gamma, r, 1),
$$

for $r \in N$. After some algebraic manipulation, the mean and variance of the EEG distribution are given, respectively, by

$$
\begin{aligned}
E(X \mid \gamma, \lambda) & =\frac{\gamma \log (\gamma)}{\lambda(\gamma-1)}, \\
\operatorname{Var}(X \mid \gamma, \lambda) & =\frac{\gamma}{\lambda^{2}}\left(\frac{2 L_{2}(1-\gamma)}{(1-\gamma)}-\frac{\gamma \log ^{2}(\gamma)}{(1-\gamma)^{2}}\right),
\end{aligned}
$$

where $L_{2}(z)$ is the dilogarithm function given by

$$
\begin{aligned}
L_{2}(z) & =\sum_{k=1}^{\infty} \frac{z^{k}}{k^{2}}=-\int_{0}^{z} \frac{\log (1-t)}{t} d t \\
& =-\int_{0}^{1} \frac{\log (1-z t)}{t} d t .
\end{aligned}
$$

The mode and the median of the EEG distribution are

$$
\begin{aligned}
\operatorname{Mode}(X \mid \gamma, \lambda) & = \begin{cases}0 & \text { if } \gamma \leq 2 \\
\frac{\log (\gamma-1)}{\lambda} & \text { if } \gamma \geq 2,\end{cases} \\
\operatorname{Median}(X \mid \gamma, \lambda) & =\frac{\log (1+\gamma)}{\lambda} .
\end{aligned}
$$

From Marshall and Olkin [16], we have the following inequality:

$$
\begin{aligned}
\operatorname{Mode}(X \mid \gamma, \lambda) & \leq \operatorname{Median}(X \mid \gamma, \lambda) \leq \frac{\gamma}{\lambda} \\
& \leq E(X \mid \gamma, \lambda)
\end{aligned}
$$

where $\lim _{\gamma \rightarrow \infty} \operatorname{Mode}(X \mid \gamma, \lambda) / E(X \mid \gamma, \lambda)=1$ 


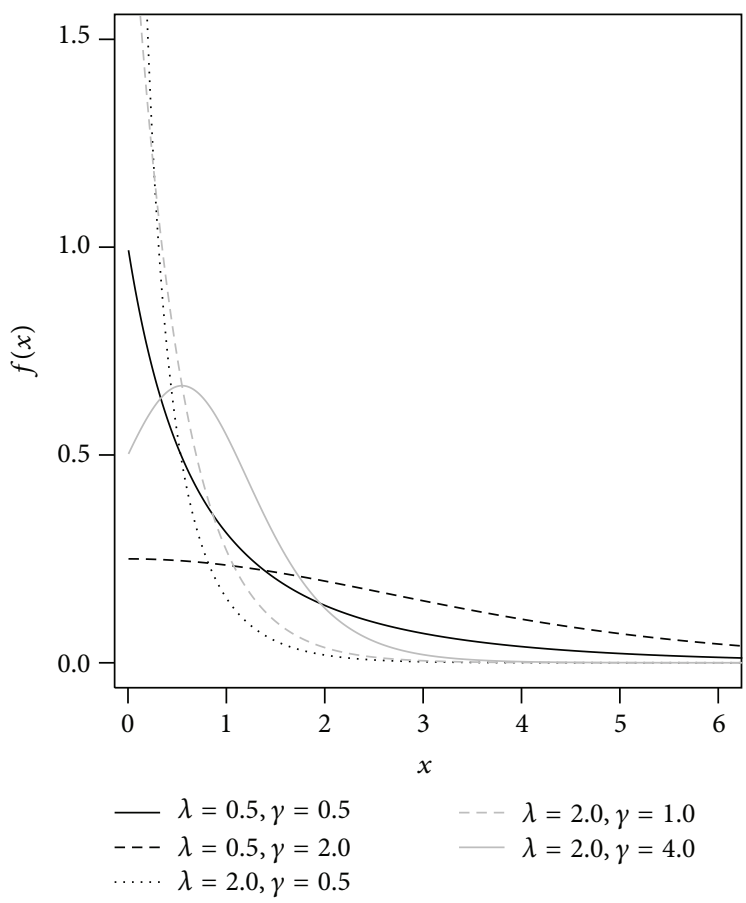

(a)

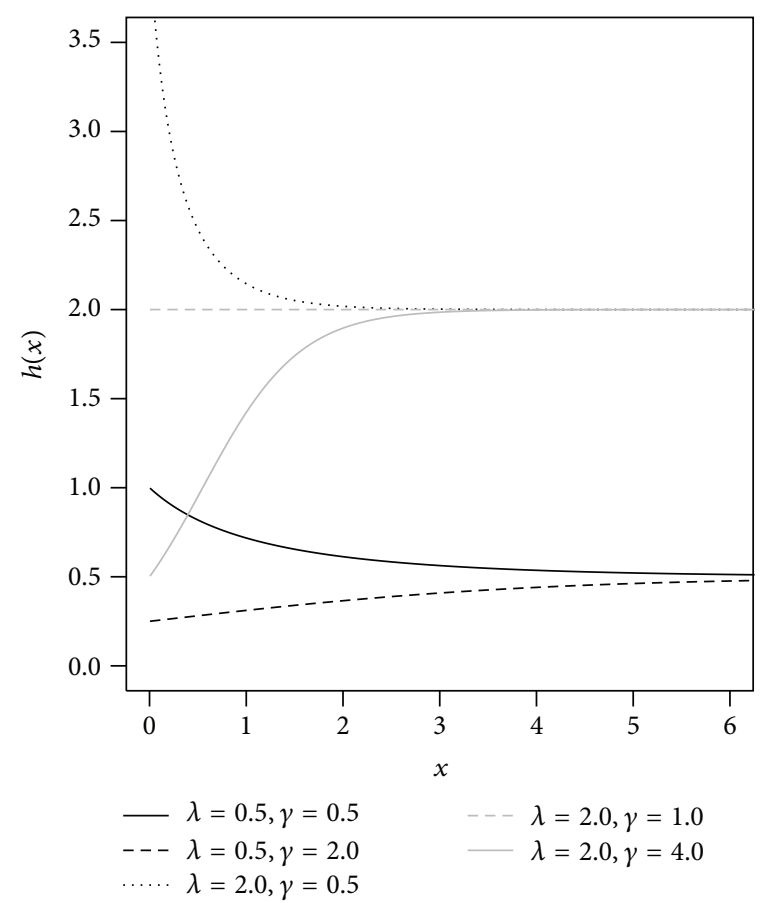

(b)

FIGURE 1: (a) Probability density function of the EEG distribution. (b) Hazard function of the EEG distribution.

Shannon's Entropy from EEG distribution [9], which played a central role as a measure of the uncertainty associated with a random variable, is given by

$$
\begin{aligned}
H_{S}(\phi, \lambda, \alpha)= & \log (\gamma \lambda) \\
& +\frac{2 \log (\gamma)-\gamma^{2} \log (\gamma) \lambda^{-1}-2 \gamma+2}{\gamma-1} .
\end{aligned}
$$

\section{Methods of Estimation}

In this section, we discuss ten different estimation methods to obtain the estimates of the parameters $\gamma$ and $\lambda$ of the EEG distribution.

3.1. Maximum Likelihood Estimation. Among the statistical inference methods, the maximum likelihood method is widely used due its desirable properties including consistency, asymptotic efficiency, and invariance. Under the maximum likelihood method, the estimators are obtained from maximizing the likelihood function (see e.g., [17]). Let $T_{1}, \ldots, T_{n}$ be a random sample such that $T \sim \operatorname{EEG}(\gamma, \lambda)$; the likelihood function from (1) is given by

$$
\begin{aligned}
& L(\gamma, \lambda ; x)=\prod_{i=1}^{n} f\left(x_{i}, \gamma, \lambda\right) \\
& \quad=(\lambda \gamma)^{n} \exp \left(-\lambda \sum_{i=1}^{n} x_{i}\right) \prod_{i=1}^{n}\left(\left(1-(1-\gamma) e^{-\lambda x_{i}}\right)^{-2}\right) .
\end{aligned}
$$

The logarithm of the likelihood function (11) is given by

$$
\begin{aligned}
l(\gamma, \lambda \mid x)= & n \log (\lambda \gamma) \\
& -\lambda \sum_{i=1}^{n} x_{i}-2 \sum_{i=1}^{n} \log \left(1-(1-\gamma) e^{-\lambda x_{i}}\right) .
\end{aligned}
$$

From $\partial l(\gamma, \lambda \mid \mathbf{t}) / \partial \gamma=0$ and $\partial l(\gamma, \lambda \mid \mathbf{t}) / \partial \lambda=0$, we get the likelihood equations

$$
\begin{array}{r}
\frac{n}{\lambda}-\sum_{i=1}^{n} x_{i}-2(1-\lambda) \sum_{i=1}^{n} \frac{x_{i} e^{-\lambda x_{i}}}{1-(1-\gamma) e^{-\lambda x_{i}}}=0, \\
\frac{n}{\lambda}-2 \sum_{i=1}^{n} \frac{e^{-\lambda x_{i}}}{1-(1-\gamma) e^{-\lambda x_{i}}}=0,
\end{array}
$$

whose solutions provide the maximum likelihood estimates, hereafter, $\gamma_{\mathrm{MLE}}$ and $\lambda_{\mathrm{MLE}}$. Numerical methods such as Newton-Raphson are required to find the solution of the nonlinear system.

For large sample sizes, the obtained estimators are not biased and asymptotically efficient. The MLE estimates are asymptotically normally distributed with joint bivariate normal distribution given by

$$
\begin{aligned}
\left(\widehat{\gamma}_{\mathrm{MLE}}, \widehat{\lambda}_{\mathrm{MLE}}\right) \sim N_{2}\left[(\gamma, \lambda), I^{-1}(\gamma, \lambda)\right] & \\
& \text { for } n \longrightarrow \infty,
\end{aligned}
$$

where $I(\gamma, \lambda)$ is the Fisher information matrix given by (see [14]) 


$$
\begin{aligned}
I(\gamma, \lambda)=\left[\begin{array}{cc}
I_{11}(\gamma, \lambda) & \frac{n\left(\gamma-1-\gamma^{2} \log (\gamma)\right)}{3 \gamma \lambda(\gamma-1)^{2}} \\
\frac{n\left(\gamma-1-\gamma^{2} \log (\gamma)\right)}{3 \gamma \lambda(\gamma-1)^{2}} & \frac{n}{3 \gamma^{2}}
\end{array}\right], \\
I_{11}(\gamma, \lambda)= \begin{cases}\frac{n\left(3(1-\gamma)-2\left((1-\gamma)-\gamma L_{2}(1-\gamma)\right)\right)}{3 \lambda^{2}(1-\gamma)} & \text { if } 0<\gamma<1 \\
\frac{n}{3 \lambda^{2}(1-\gamma)}\left(1-\gamma\left(1+\frac{\pi^{2}}{3}+\log ^{2}(\gamma)-2 L_{2}\left(\frac{1}{\gamma}\right)\right)\right), & \text { if } \gamma>1 .\end{cases}
\end{aligned}
$$

3.2. Moments Estimators. The method of moments is one of the oldest procedures used for estimating parameters in statistical models. The moment estimators (ME) of the EEG distribution can be obtained by equating the first two theoretical moments,

$$
\begin{aligned}
& \frac{1}{n} \sum_{i=1}^{n} x_{i}=\frac{\gamma \log (\gamma)}{\lambda(\gamma-1)}, \\
& \frac{1}{n} \sum_{i=1}^{n} x_{i}^{2}=\frac{2 \gamma L_{2}(1-\gamma)}{\lambda^{2}(1-\gamma)},
\end{aligned}
$$

with the sample moments $\bar{x}=(1 / n) \sum_{i=1}^{n} x_{i}$ and $(1 / n) \sum_{i=1}^{n} x_{i}^{2}$, respectively. After some algebraic manipulation, the estimate for $\widehat{\lambda}_{\mathrm{ME}}$ can be obtained by solving

$$
\widehat{\lambda}_{\mathrm{ME}}=\frac{\gamma \log (\gamma)}{\bar{x}(\gamma-1)} .
$$

Note that, by substituting $\widehat{\lambda}_{\mathrm{ME}}$ in (18), the estimate for $\widehat{\gamma}_{\mathrm{ME}}$ can be obtained by solving

$$
\frac{2(1-\gamma) L_{2}(1-\gamma) \bar{x}^{2}}{\gamma \log (\gamma)^{2}}-\frac{1}{n} \sum_{i=1}^{n} x_{i}^{2}=0 .
$$

Therefore, we firstly compute $\widehat{\gamma}_{\mathrm{ME}}$ and, by substituting such estimate in (19), the estimate $\widehat{\lambda}_{\mathrm{ME}}$ is obtained.

3.3. Method of Modified Moments. A simple modification can be made in the method of moments for estimating the parameters of the EEG distribution. To obtain the moment estimators (MME), consider that

$$
\begin{aligned}
E(X \mid \gamma, \lambda) & =\frac{\gamma \log (\gamma)}{\lambda(\gamma-1)} \\
\operatorname{Var}(X \mid \gamma, \lambda) & =\frac{\gamma}{\lambda^{2}}\left(\frac{2 L_{2}(1-\gamma)}{(1-\gamma)}-\frac{\gamma \log ^{2}(\gamma)}{(1-\gamma)^{2}}\right) .
\end{aligned}
$$

Note that the population coefficient of variation given by

$$
\begin{aligned}
\operatorname{CV}(X \mid \gamma, \lambda) & =\frac{\sqrt{\operatorname{Var}(X \mid \gamma, \lambda)}}{E(X \mid \gamma, \lambda)} \\
& =\sqrt{\frac{2\left(\gamma^{-1}-1\right) L_{2}(1-\gamma)}{\log ^{2}(\gamma)}-1}
\end{aligned}
$$

is independent of the scale parameter $\lambda$. So the estimate for $\widehat{\gamma}_{\mathrm{ME}}$ can be obtained by solving the nonlinear equation

$$
\sqrt{\frac{2\left(\gamma^{-1}-1\right) L_{2}(1-\gamma)}{\log ^{2}(\gamma)}-1}-\frac{s}{\bar{x}}=0
$$

and, by substituting $\widehat{\gamma}_{\mathrm{MME}}$ in (23), the estimate $\widehat{\lambda}_{\mathrm{MME}}$ for $\lambda$ can be obtained by solving

$$
\widehat{\lambda}_{\mathrm{MME}}=\frac{\gamma \log (\gamma)}{\bar{x}(\gamma-1)}
$$

3.4. Percentile Estimators. The percentile estimator, originally suggested by Kao $[18,19]$, is a statistical method used to estimate the unknown parameters by comparing the sample points with the theoretical ones. This method has been widely used in distributions that have the quantile function in a closed form, such as the Weibull distribution and the Generalized Exponential distribution. For the EEG distribution, the quantile function is given by

$$
Q(p \mid \gamma, \lambda)=\frac{1}{\lambda} \log \left(\frac{1-(1-\gamma) p}{1-p}\right) .
$$

The percentile estimates (PCE), $\widehat{\gamma}_{\mathrm{PCE}}$ and $\hat{\lambda}_{\mathrm{PCE}}$, can be obtained by minimizing

$$
\sum_{i=1}^{n}\left(x_{(i)}-\frac{1}{\lambda} \log \left(\frac{1-(1-\gamma) p}{1-p}\right)\right)^{2},
$$


with respect to $\gamma$ and $\lambda$, where $p_{i}$ denotes some estimate of $F\left(x_{(i)} ; \gamma, \lambda\right)$. The estimates of $\gamma$ and $\lambda$ can also be obtained by solving the following nonlinear equations:

$$
\begin{aligned}
\sum_{i=1}^{n} & {\left[x_{i}-\frac{1}{\lambda} \log \left(\frac{1-(1-\gamma) p}{1-p}\right)\right]\left(\frac{p}{1-(1-\gamma) p}\right) } \\
& =0, \\
\sum_{i=1}^{n} & {\left[x_{i}-\frac{1}{\lambda} \log \left(\frac{1-(1-\gamma) p}{1-p}\right)\right] } \\
& \cdot\left(\frac{1}{\lambda^{2}} \log \left(\frac{1-(1-\gamma) p}{1-p}\right)\right)=0,
\end{aligned}
$$

respectively. In this paper, we consider $p_{i}=i /(n+1)$. However, several estimators of $p_{i}$ can be used instead (see [20]).

3.5. L-Moments Estimators. Hosking [21] proposed an alternative method of estimation analogous to conventional moments, namely, $L$-moments estimators. These estimators are obtained by equating the sample $L$-moments with the population $L$-moments. Hosking [21] states that the $L$-moment estimators are more robust than the usual moment estimators and are also relatively robust to the effects of outliers and reasonably efficient when compared to the MLE for some distributions.

For the EEG distribution, the $L$-moments estimators (LME) can be obtained by equating the first two sample $L$ moments with the corresponding population $L$-moments. The first two sample $L$-moments are

$$
\begin{aligned}
& l_{1}=\frac{1}{n} \sum_{i=1}^{n} x_{(i)}, \\
& l_{2}=\frac{2}{n(n-1)} \sum_{i=1}^{n}(i-1) x_{(i)}-l_{1},
\end{aligned}
$$

and the first two population $L$-moments are

$$
\begin{aligned}
\mu_{1}(\gamma, \lambda) & =\int_{0}^{1} Q(p \mid \gamma, \lambda) d p=E(X \mid \gamma, \lambda) \\
& =\frac{\gamma \log (\gamma)}{\lambda(\gamma-1)}, \\
\mu_{2}(\gamma, \lambda) & =\int_{0}^{1} Q(p \mid \gamma, \lambda)(2 p-1) d p \\
& =\frac{1}{\lambda}\left(\frac{\gamma^{2}-2 \gamma \log (\gamma)-1}{2(\gamma-1)^{2}}+\frac{1}{2}\right),
\end{aligned}
$$

where $Q(p \mid \gamma, \lambda)$ is given in (25). After some algebraic manipulations, the estimate for $\widehat{\gamma}_{\text {LME }}$ can be obtained by solving the nonlinear equation

$$
\frac{\bar{x}}{1-\gamma}+\frac{\bar{x}}{\log (\gamma)}-l_{2}=0 .
$$

Note that, by substituting $\widehat{\gamma}_{\text {LME }}$ in (30), the estimate for $\widehat{\lambda}_{\mathrm{LME}}$ can be obtained by solving

$$
\hat{\lambda}_{\mathrm{LME}}=\frac{\widehat{\gamma}_{\mathrm{LME}} \log \left(\widehat{\gamma}_{\mathrm{LME}}\right)}{\bar{x}\left(\widehat{\gamma}_{\mathrm{LME}}-1\right)} .
$$

3.6. Ordinary and Weighted Least Squares Estimates. Let $t_{(1)}, t_{(2)}, \ldots, t_{(n)}$ denote the order statistics (we assume the same notation for the next subsections) of the random sample of size $n$ from a distribution function $F(\mathbf{x} \mid \gamma, \lambda)$. The least square estimators (LSE) $\widehat{\gamma}_{\text {LSE }}$ and $\hat{\lambda}_{\text {LSE }}$ can be obtained by minimizing

$$
S(\gamma, \lambda)=\sum_{i=1}^{n}\left[F\left(x_{(i)} \mid \gamma, \lambda\right)-\frac{i}{n+1}\right]^{2},
$$

with respect to $\gamma$ and $\lambda$, where $F(\mathbf{t} \mid \gamma, \lambda)$ is given by (2). Equivalently, they can be obtained by solving the following nonlinear equations:

$$
\begin{aligned}
& \sum_{i=1}^{n}\left[F\left(x_{(i)} \mid \gamma, \lambda\right)-\frac{i}{n+1}\right] \Delta_{1}\left(x_{(i)} \mid \gamma, \lambda\right)=0, \\
& \sum_{i=1}^{n}\left[F\left(x_{(i)} \mid \gamma, \lambda\right)-\frac{i}{n+1}\right] \Delta_{2}\left(x_{(i)} \mid \gamma, \lambda\right)=0,
\end{aligned}
$$

where

$$
\begin{aligned}
& \Delta_{1}\left(x_{(i)} \mid \gamma, \lambda\right)=\frac{e^{\lambda x_{(i)}}-1}{\left(e^{\lambda x_{(i)}}-1+\gamma\right)^{2}}, \\
& \Delta_{2}\left(x_{(i)} \mid \gamma, \lambda\right)=\frac{\lambda x e^{\lambda x_{(i)}}}{\left(e^{\lambda x_{(i)}}-1+\gamma\right)^{2}} .
\end{aligned}
$$

The weighted least squares estimates (WLSE), $\widehat{\gamma}_{\text {WLSE }}$ and $\widehat{\lambda}_{\text {WLSE }}$, can be obtained by minimizing

$$
\begin{aligned}
& W(\gamma, \lambda) \\
& \quad=\sum_{i=1}^{n} \frac{(n+1)^{2}(n+2)}{i(n-i+1)}\left[F\left(t_{(i)} \mid \gamma, \lambda\right)-\frac{i}{n+1}\right]^{2} .
\end{aligned}
$$

These estimates can also be obtained by solving the following nonlinear equations:

$$
\begin{aligned}
& \sum_{i=1}^{n} \frac{(n+1)^{2}(n+2)}{i(n-i+1)}\left[F\left(x_{(i)} \mid \gamma, \lambda\right)-\frac{i}{n+1}\right] \\
& \cdot \Delta_{1}\left(x_{(i)} \mid \gamma, \lambda\right)=0, \\
& \sum_{i=1}^{n} \frac{(n+1)^{2}(n+2)}{i(n-i+1)}\left[F\left(x_{(i)} \mid \gamma, \lambda\right)-\frac{i}{n+1}\right] \\
& \cdot \Delta_{2}\left(x_{(i)} \mid \gamma, \lambda\right)=0 .
\end{aligned}
$$

3.7. Method of Maximum Product of Spacings. The maximum product of spacings (MPS) method is a powerful alternative 
to MLE for the estimation of the unknown parameters of continuous univariate distributions. Proposed by Cheng and Amin [22, 23], this method was also independently developed by Ranneby [24] as approximation to the Kullback-Leibler measure of information.

Let $D_{i}(\gamma, \lambda)=F\left(x_{(i)} \mid \gamma, \lambda\right)-F\left(x_{(i-1)} \mid \gamma, \lambda\right)$, for $i=$ $1,2, \ldots, n+1$, be the uniform spacings of a random sample from the EEG distribution, where $F\left(x_{(0)} \mid \gamma, \lambda\right)=0$ and $F\left(x_{(n+1)} \mid \gamma, \lambda\right)=1$. Clearly $\sum_{i=1}^{n+1} D_{i}(\gamma, \lambda)=1$. The maximum product of spacings estimates, $\widehat{\gamma}_{\text {MPS }}$ and $\widehat{\lambda}_{\text {MPS }}$, are obtained by maximizing the geometric mean of the spacings,

$$
G(\gamma, \lambda)=\left[\prod_{i=1}^{n+1} D_{i}(\gamma, \lambda)\right]^{1 /(n+1)}
$$

with respect to $\gamma$ and $\lambda$, or, equivalently, by maximizing the logarithm of the geometric mean of sample spacings:

$$
H(\gamma, \lambda)=\frac{1}{n+1} \sum_{i=1}^{n+1} \log D_{i}(\gamma, \lambda)
$$

The estimates $\widehat{\gamma}_{\text {MPS }}$ and $\widehat{\lambda}_{\text {MPS }}$ of the parameters $\gamma$ and $\lambda$ can be obtained by solving the following nonlinear equations

$$
\begin{aligned}
& \frac{\partial H(\gamma, \lambda)}{\partial \gamma}=\frac{1}{n+1} \\
& \quad \cdot \sum_{i=1}^{n+1} \frac{1}{D_{i}(\gamma, \lambda)}\left[\Delta_{1}\left(x_{(i)} \mid \gamma, \lambda\right)-\Delta_{1}\left(x_{(i-1)} \mid \gamma, \lambda\right)\right] \\
& \quad=0, \\
& \frac{\partial H(\gamma, \lambda)}{\partial \lambda}=\frac{1}{n+1} \\
& \quad \cdot \sum_{i=1}^{n+1} \frac{1}{D_{i}(\gamma, \lambda)}\left[\Delta_{2}\left(x_{(i)} \mid \gamma, \lambda\right)-\Delta_{2}\left(x_{(i-1)} \mid \gamma, \lambda\right)\right] \\
& =0,
\end{aligned}
$$

where $\Delta_{1}(\cdot \mid \gamma, \lambda)$ and $\Delta_{2}(\cdot \mid \gamma, \lambda)$ are given in (35).

Note that if $x_{(i+k)}=x_{(i+k-1)}=\cdots=x_{(i)}$ we get $D_{i+k}(\gamma, \lambda)=D_{i+k-1}(\gamma, \lambda)=\cdots=D_{i}(\gamma, \lambda)=0$. Therefore, the MPS estimators are sensitive to closely spaced observations, especially ties. When the ties are due to multiple observations, $D_{i}(\gamma, \lambda)$ should be replaced by the corresponding likelihood $f\left(x_{(i)}, \gamma, \lambda\right)$, since $x_{(i)}=x_{(i-1)}$.

Cheng and Amin [23] proved desirable properties of the MPS such as asymptotic efficiency and invariance; they also proved that the consistency of maximum product of spacings estimators holds under much more general conditions than for maximum likelihood estimators. The authors also present an interesting proof that the MPS estimates converge asymptotically to the ML estimates. Therefore, for the EEG distribution, the MPS estimators are asymptotically normally distributed (see [25] for more details) with joint bivariate normal distribution given by

$$
\begin{aligned}
&\left(\widehat{\gamma}_{\mathrm{MPS}}, \widehat{\lambda}_{\mathrm{MPS}}\right) \sim N_{2}\left[(\gamma, \lambda), I^{-1}(\gamma, \lambda)\right] \\
& \text { for } n \longrightarrow \infty,
\end{aligned}
$$

where $I(\gamma, \lambda)$ is the Fisher information matrix.

3.8. The Cramer-von Mises Minimum Distance Estimators. The Cramer-von Mises estimator (CME) is a type of minimum distance estimators (also called maximum goodnessof-fit estimators) which is based on the difference between the estimate of the cumulative distribution function and the empirical distribution function (see, $[26,27])$.

MacDonald [28] motivates the choice of Cramer-von Mises type minimum distance estimators providing empirical evidence that the bias of the estimator is smaller than the other minimum distance estimators. The Cramer-von Mises estimates, $\widehat{\gamma}_{\mathrm{CME}}$ and $\widehat{\lambda}_{\mathrm{CME}}$, are obtained by minimizing

$$
C(\gamma, \lambda)=\frac{1}{12 n}+\sum_{i=1}^{n}\left(F\left(x_{(i)} \mid \gamma, \lambda\right)-\frac{2 i-1}{2 n}\right)^{2},
$$

with respect to $\gamma$ and $\lambda$. These estimates can also be obtained by solving the following nonlinear equations:

$$
\begin{aligned}
& \sum_{i=1}^{n}\left(F\left(x_{(i)} \mid \gamma, \lambda\right)-\frac{2 i-1}{2 n}\right) \Delta_{1}\left(x_{(i)} \mid \gamma, \lambda\right)=0, \\
& \sum_{i=1}^{n}\left(F\left(x_{(i)} \mid \gamma, \lambda\right)-\frac{2 i-1}{2 n}\right) \Delta_{2}\left(x_{(i)} \mid \gamma, \lambda\right)=0,
\end{aligned}
$$

where $\Delta_{1}(\cdot \mid \gamma, \lambda)$ and $\Delta_{2}(\cdot \mid \gamma, \lambda)$ are given in (35).

3.9. Methods of Anderson-Darling. Another type of minimum distance estimators is based on Anderson-Darling statistic (see [27]) and is known as the Anderson-Darling estimator (ADE). The Anderson-Darling estimates, $\widehat{\gamma}_{\mathrm{ADE}}$ and $\hat{\lambda}_{\mathrm{ADE}}$, of the parameters $\gamma$ and $\lambda$ are obtained by minimizing, with respect to $\gamma$ and $\lambda$, the function

$$
\begin{aligned}
& A(\gamma, \lambda)=-n-\frac{1}{n} \sum_{i=1}^{n}(2 i-1) \\
& \quad \cdot\left(\log F\left(x_{(i)} \mid \gamma, \lambda\right)+\log S\left(x_{(n+1-i)} \mid \gamma, \lambda\right)\right) .
\end{aligned}
$$

These estimates can also be obtained by solving the following nonlinear equations:

$$
\begin{aligned}
& \sum_{i=1}^{n}(2 i-1)\left[\frac{\Delta_{1}\left(x_{(i)} \mid \gamma, \lambda\right)}{F\left(x_{(i)} \mid \gamma, \lambda\right)}-\frac{\Delta_{1}\left(x_{(n+1-i)} \mid \gamma, \lambda\right)}{S\left(x_{(n+1-i)} \mid \gamma, \lambda\right)}\right] \\
& \quad=0, \\
& \sum_{i=1}^{n}(2 i-1)\left[\frac{\Delta_{2}\left(x_{(i)} \mid \gamma, \lambda\right)}{F\left(x_{(i)} \mid \gamma, \lambda\right)}-\frac{\Delta_{2}\left(x_{(n+1-i)} \mid \gamma, \lambda\right)}{S\left(x_{(n+1-i)} \mid \gamma, \lambda\right)}\right] \\
& \quad=0,
\end{aligned}
$$

where $\Delta_{1}(\cdot \mid \gamma, \lambda)$ and $\Delta_{2}(\cdot \mid \gamma, \lambda)$ are in (35). 


\section{Simulation Study}

In this section, we develop a simulation study via Monte Carlo methods. The main goal of these simulations is to compare the efficiency of the different estimation methods for the parameters of the EEG distribution. The following procedure was adopted:

(1) Set the sample size $n$ and the vector of parameter values $\boldsymbol{\theta}=(\lambda, \gamma)$.

(2) Generate values of $\operatorname{EEG}(\lambda, \gamma)$ with size $n$.

(3) Using the values obtained in step (2), compute $\hat{\lambda}$ and $\widehat{\gamma}$ via MLE, ME, MME, LSE, WLSE, PCE, MPS, CME, and ADE.

(4) Repeat steps (2) and (3) $N$ times.

(5) Using $\widehat{\boldsymbol{\theta}}$ and $\boldsymbol{\theta}$, compute the mean relative estimates (MRE) $\sum_{j=1}^{N}\left(\left(\widehat{\theta}_{i, j} / \theta_{i}\right) / N\right)$ and the mean square errors (MSE) $\sum_{j=1}^{N}\left(\left(\widehat{\theta}_{i, j}-\theta_{i}\right)^{2} / N\right), i=1,2$.

We expect that, considering this approach, the MREs are closer to one with smaller MSEs. The results were computed using the software R (R Core Development Team). The seed used to generate the random values was 2015 . The chosen values to perform this procedure were $\boldsymbol{\theta}=((0.5,2),(2,4))$, $N=10,000$, and $n=(15,20,25, \ldots, 130)$. The values of $\boldsymbol{\theta}$ were selected to allow, respectively, the decreasing and increasing shape in the hazard function. Another motivation comes from the fact that, for $\boldsymbol{\theta}=(0.5,2)$, we have analogous results for the exponential geometric distribution [2] and, for $\boldsymbol{\theta}=(2,4)$, the results are analogous for the Complementary Exponential Geometric distribution [4].

Figures 2 and 3 present the MREs and MSEs for the estimates of $\boldsymbol{\theta}$ for $N$ simulated samples considering different values of $n$. The horizontal lines in Figures 2 and 3 correspond to MREs and MSEs being, respectively, one and zero.

It is worth noting that we only considered the samples in which all estimation procedures had converged, getting at the end $N$ simulated samples for different values of $n$. Figure 4 presents the proportion of failure from each method.

Based on these figures, the MSEs of all estimates tend to zero for large $n$ and also, as expected, the values of MREs tend to one; that is, the estimates are asymptotically unbiased for the parameters. The ME and the CME estimators have, respectively, the largest MREs and MSEs among all the considered estimators. The percentile and the LSE estimators have, respectively, the largest proportion of failure for estimating the parameters of the EEG distribution.

The MPS estimators have the smallest MSEs and the MREs nearest to one for both parameters proving to be the most efficient procedure for estimating the unknown parameters. Moreover, the MPS estimators have good theoretical properties [23] such as consistency, asymptotic efficiency, normality, and invariance. Therefore, we conclude that the MPS estimators should be used for estimating the parameters of the EEG distribution.
TABLE 1: Data set related to the ages of 18 patients who died from other causes than cancer.

\begin{tabular}{ccccccccc}
\hline 0.3 & 4 & 7.4 & 15.5 & 23.4 & 46 & 46 & 51 & 65 \\
68 & 83 & 88 & 96 & 110 & 111 & 112 & 132 & 162 \\
\hline
\end{tabular}

\section{Applications}

In this section, we considered two real data sets. The first one is presented by Boag [29] and is related to the ages (in months) of 18 patients who died from other causes than cancer. The second data set is presented by Silva [30] and refers to the serum-reversal time (in days) of 143 children born to HIV-infected mothers who did not receive anti-HIV treatment (Table 4).

In Section 4, our simulation study indicated that the MPS estimators should be used for estimating the parameters of the EEG distribution. Initially, we compared the estimates obtained from the different procedures with the MPS estimator in terms of MREs. Then, we compared the results obtained from the EEG distribution fitted by the MPS estimators with some common lifetime models, such as Weibull, Gamma, Lognormal, and Generalized Exponential distributions.

The Kolmogorov-Smirnov (KS) test is considered to check the goodness of fit. This procedure is based on the KS statistic $D_{n}=\sup _{x}\left|F_{n}(x)-F(x ; \theta, \lambda)\right|$, where sup $x$ is the supremum of the set of distances, $F_{n}(x)$ is the empirical distribution function, and $F(x ; \theta, \lambda)$ is cumulative distribution function. In this case, we test the null hypothesis that the data comes from $F(x ; \theta, \lambda)$, and, with significance level of $5 \%$, we will reject the null hypothesis if $p$ value is smaller than 0.05. As discrimination criterion method, we considered the AIC (Akaike Information Criteria), AICc (Corrected Akaike Information Criteria), HQIC (Hannan-Quinn Information Criteria), and the CAIC (Consistent Akaike Information Criteria) computed, respectively, by $\mathrm{AIC}=-2 l(\widehat{\Theta}, x)+2 k$, $\mathrm{AICc}=\mathrm{AIC}+2 k(k+1) /(n-k-1), \mathrm{HQIC}=-2 l(\widehat{\Theta}, x)+$ $2 k \log (\log (n))$, and CAIC $=-2 l(\widehat{\Theta}, x)+k \log (n)+1$, where $k$ is the number of parameters to be fitted and $\widehat{\Theta}$ is the estimate of $\Theta$. Given a set of candidate models for $t$, the preferred model is the one which provides the minimum values.

5.1. Boag Data Set. Table 1 presents the data set related to the ages (in months) of 18 patients who died from other causes than cancer extracted from Boag [29], which considered the Lognormal distribution to describe such data.

Considering the MPS estimators, we obtain $\widehat{\lambda}_{\mathrm{MPS}}=$ 0.02101 and $\mathrm{CI}_{95 \%}(\lambda)=(0.00618 ; 0.03583)$ and $\widehat{\gamma}_{\mathrm{MPS}}=$ 2.46430 and $\mathrm{CI}_{95 \%}(\gamma)=(0.00000 ; 6.28060)$. In Table 2 , we compared the estimates obtained from the different procedures with the MPS estimator in terms of MREs.

Table 2 confirmed the results obtained from our simulation study, in which for small sample sizes the obtained results may differ depending on the estimation procedure. For example, considering the method of moments, the estimate for $\gamma$ is $52 \%$ smaller than $\widehat{\gamma}_{\text {MPS }}$. Table 3 presents the results from KS test ( $p$ value), AIC, AICc, HQIC, and CAIC, for the EEG distribution adjusted by the MPS procedure and 


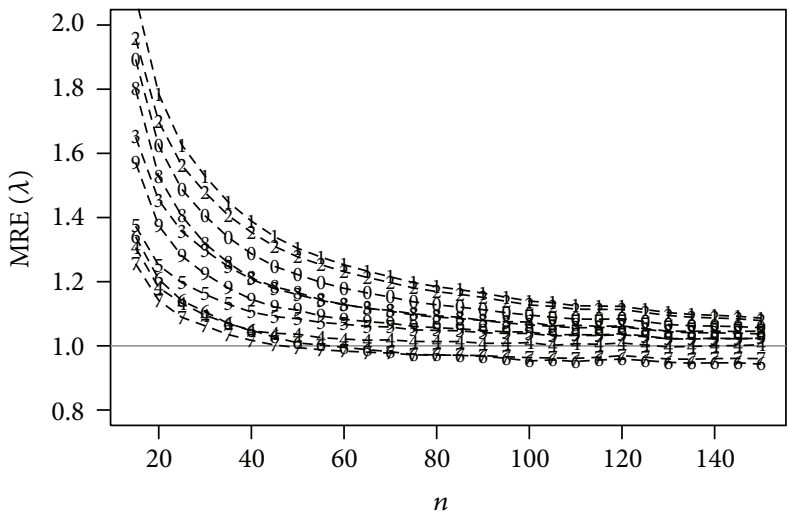

(a)

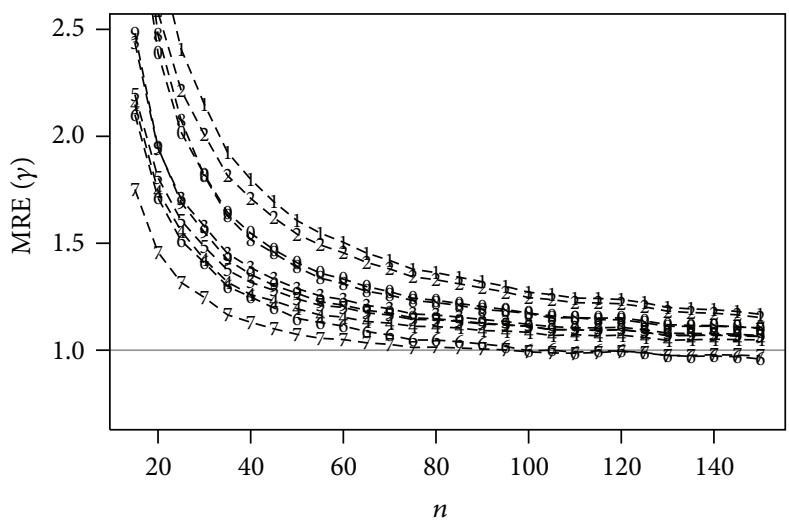

(c)

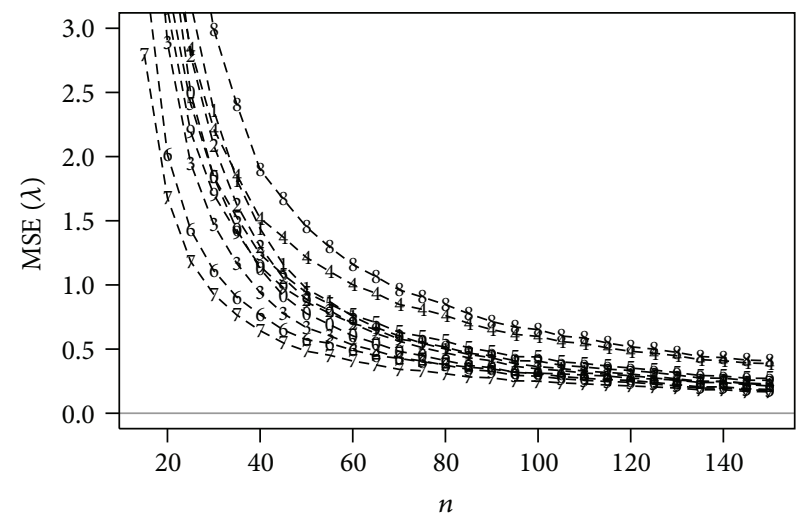

(b)

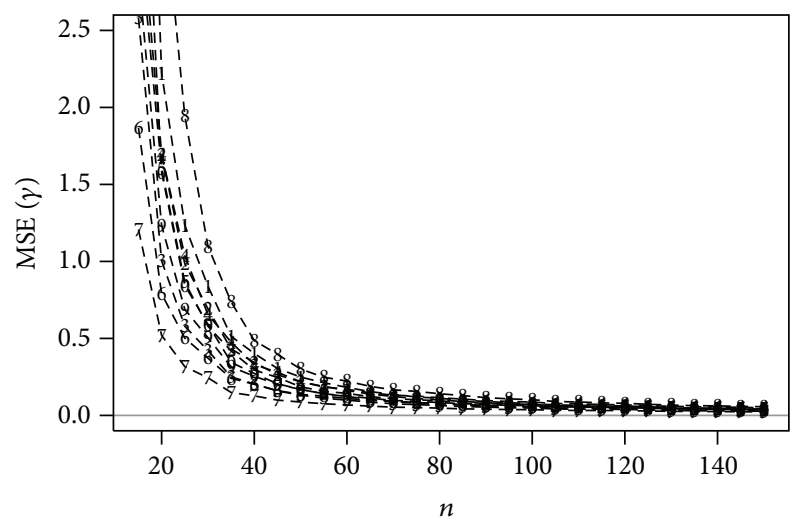

(d)

FIGURE 2: MREs and MSEs related from the estimates of $\lambda=2$ and $\gamma=0.5$ for $N$ simulated samples, considering different values of $n$ obtained using the following estimation methods: (1) MLE, (2) ME, (3) MME, (4) LME, (5) LSE, (6) WLSE, (7) PCE, (8) MPS, (9) CME, and (10) ADE.

TABLE 2: The MREs of the estimates obtained from the different procedures compared to the MPS considering the data set related to the ages of 18 patients who died from other causes than cancer.

\begin{tabular}{lccccccccc}
\hline$\widehat{\boldsymbol{\theta}}_{\text {MPS }} / \widehat{\boldsymbol{\theta}}$ & MLE & ME & MME & LME & LSE & WLSE & PCE & CME & ADE \\
\hline$\lambda$ & 0.8033 & 0.7057 & 0.7397 & 0.8650 & 1.0365 & 0.9689 & 0.8192 & 0.8966 & 1.0112 \\
$\gamma$ & 0.6281 & 0.4883 & 0.5537 & 0.8226 & 0.9630 & 0.8908 & 0.6186 & 0.7219 & 1.0222 \\
\hline
\end{tabular}

TABle 3: Results of the KS test ( $p$ value), AIC, AICc, HQIC, and CAIC for the different probability distributions considering the data set related to the ages of 18 patients who died from other causes than cancer.

\begin{tabular}{lccccc}
\hline Test & EEG & Weibull & Gamma & Lognormal & GE \\
\hline KS & $\mathbf{0 . 9 3 0 3}$ & 0.5185 & 0.3361 & 0.0561 & 0.3156 \\
AIC & $\mathbf{1 8 9 . 8 0 9}$ & 191.447 & 191.801 & 201.695 & 191.798 \\
AICc & $\mathbf{1 9 0 . 6 0 9}$ & 192.247 & 192.601 & 202.495 & 192.598 \\
HQIC & $\mathbf{1 9 0 . 0 5 4}$ & 191.692 & 192.047 & 201.940 & 192.044 \\
CAIC & $\mathbf{1 9 3 . 5 8 9}$ & 195.227 & 195.582 & 205.475 & 195.579 \\
\hline
\end{tabular}

for different probability distributions. In Figure 5, we have the survival function adjusted by different distributions and nonparametric survival estimator.

Comparing the empirical survival function with the adjusted distributions, a better fit for the EEG distribution among the chosen models can be observed. This result is confirmed from AIC, AICc, HQIC, and CAIC, since EEG distribution has the minimum values and $p$ values returned from the KS test are greater than the chosen models. From our proposed methodology, we observe that the extended exponential geometric distribution has superior fit among the chosen models. In this case, each of the causes of the death can be described by exponential distribution; since the lifetime associated with a particular risk is not observable (latent variables), we observe only the maximum lifetime $(\gamma>1)$ value among all risks, where the number of causes follows geometric distribution.

5.2. Children Exposed to the Vertical Transmission of HIV. The data set related to the serum-reversal time (in days) of 143 children born to HIV-infected mothers is presented in Table 3. 


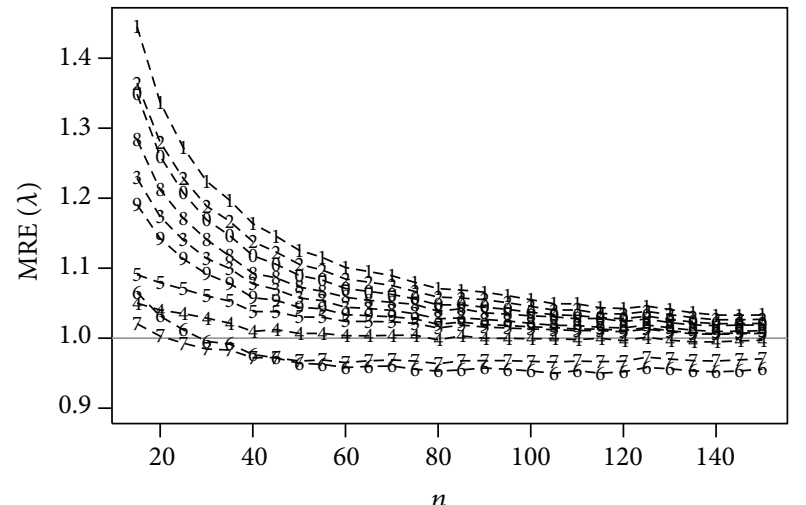

(a)

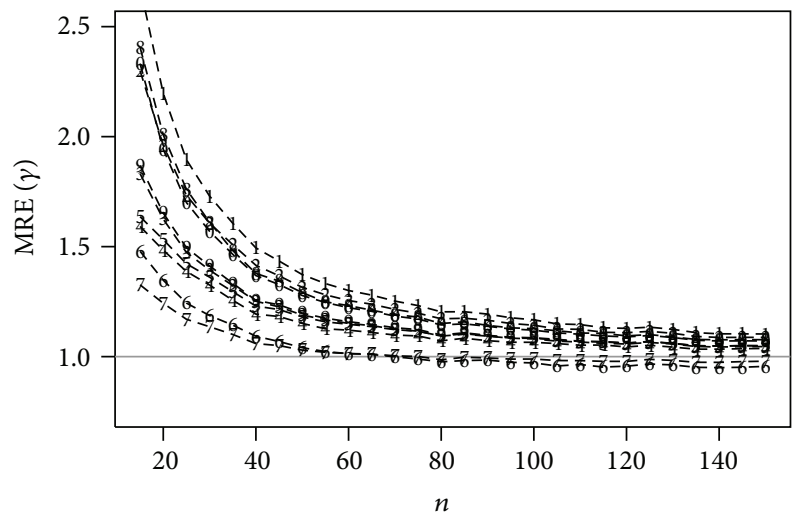

(c)

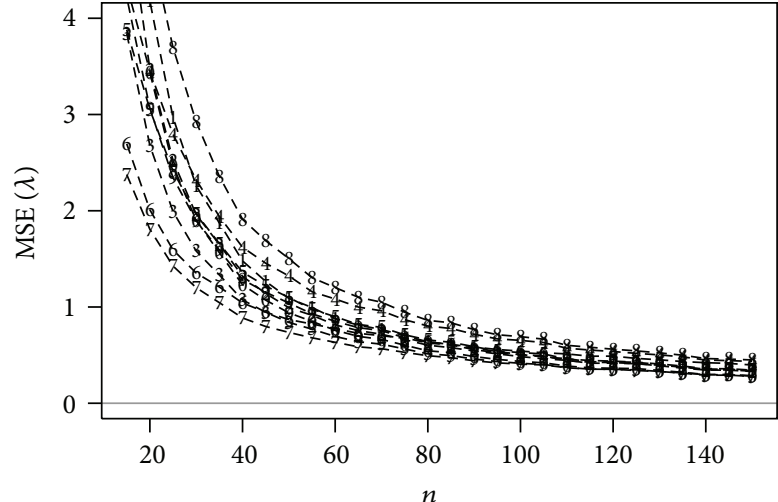

(b)

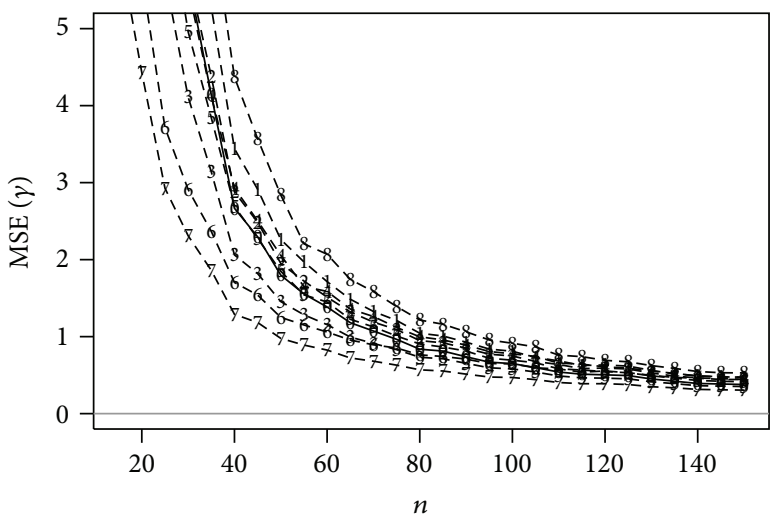

(d)

FIGURE 3: MREs and MSEs related from the estimates of $\lambda=4$ and $\gamma=2$ for $N$ simulated samples, considering different values of $n$ obtained using the following estimation methods: (1) MLE, (2) ME, (3) MME, (4) LME, (5) LSE, (6) WLSE, (7) PCE, (8) MPS, (9) CME, and (10) ADE.

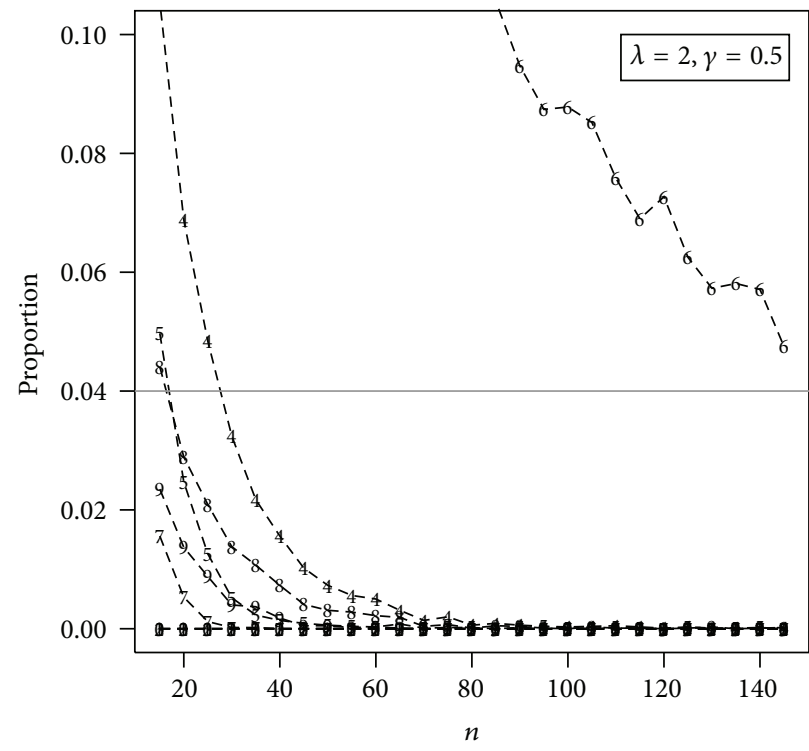

(a)

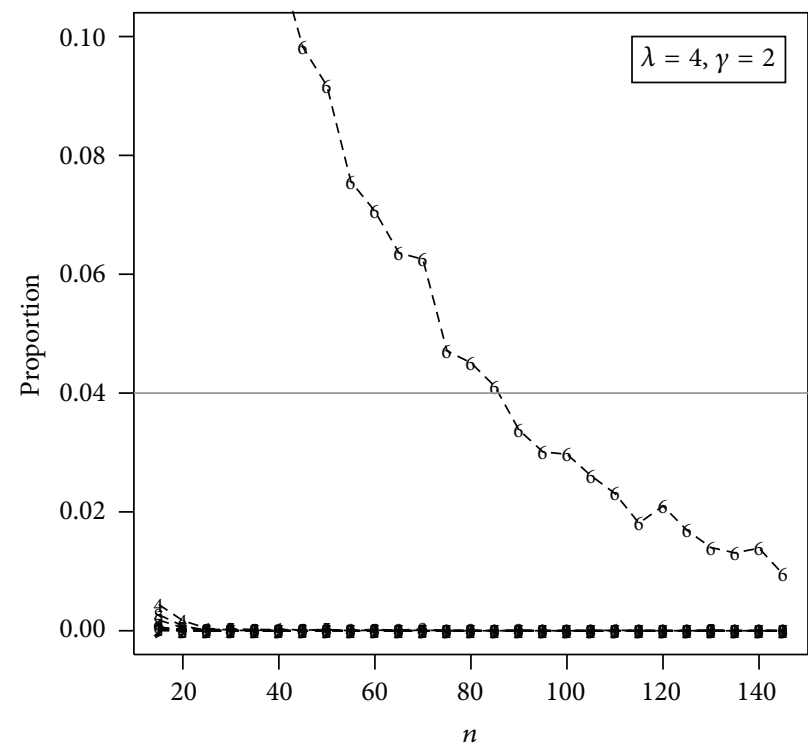

(b)

FIGURE 4: Rate of convergence considering different values of $n$ obtained using the following estimation methods: (1) MLE, (2) ME, (3) MME, (4) LME, (5) LSE, (6) WLSE, (7) PCE, (8) MPS, (9) CME, and (10) ADE. 
TABLE 4: Data set related to the serum-reversal time (in days) of 143 children born to HIV-infected mothers.

\begin{tabular}{lccccccccccccccc}
\hline 2 & 2 & 2 & 5 & 9 & 9 & 19 & 32 & 32 & 46 & 50 & 56 & 56 & 78 & 91 & 95 \\
106 & 129 & 129 & 148 & 149 & 156 & 175 & 176 & 191 & 192 & 204 & 209 & 211 & 225 & 229 & 230 \\
238 & 254 & 271 & 274 & 276 & 290 & 291 & 292 & 297 & 297 & 322 & 334 & 334 & 334 & 344 & 346 \\
353 & 353 & 359 & 365 & 366 & 367 & 370 & 378 & 378 & 382 & 382 & 385 & 398 & 400 & 402 & 414 \\
422 & 424 & 428 & 434 & 435 & 440 & 443 & 446 & 448 & 448 & 451 & 454 & 459 & 460 & 461 & 473 \\
480 & 481 & 484 & 487 & 493 & 497 & 498 & 502 & 511 & 511 & 513 & 514 & 516 & 521 & 524 & 526 \\
537 & 538 & 541 & 543 & 544 & 544 & 545 & 549 & 551 & 553 & 553 & 554 & 556 & 559 & 571 & 576 \\
577 & 578 & 582 & 588 & 590 & 596 & 609 & 610 & 615 & 619 & 626 & 627 & 648 & 653 & 678 & 680 \\
687 & 696 & 729 & 744 & 748 & 777 & 847 & 848 & 867 & 874 & 894 & 901 & 907 & 974 & 1021 & \\
\hline
\end{tabular}

TABLE 5: The MREs of the estimates obtained from the different procedures compared to the MPS considering the data set related to the serum-reversal time (in days) of 143 children born to HIV-infected mothers.

\begin{tabular}{lccccccccc}
\hline$\widehat{\boldsymbol{\theta}}_{\mathrm{MPS}} / \widehat{\boldsymbol{\theta}}$ & MLE & ME & MME & LME & LSE & WLSE & PCE & CME & ADE \\
\hline$\lambda$ & 0.9922 & 0.9726 & 0.9774 & 1.0007 & 0.9481 & 0.9587 & 0.9580 & 0.9352 \\
$\gamma$ & 0.9910 & 1.0169 & 1.0341 & 1.1184 & 0.8039 & 0.8684 & 0.9253 & 0.7682 & 1.0259 \\
\hline
\end{tabular}

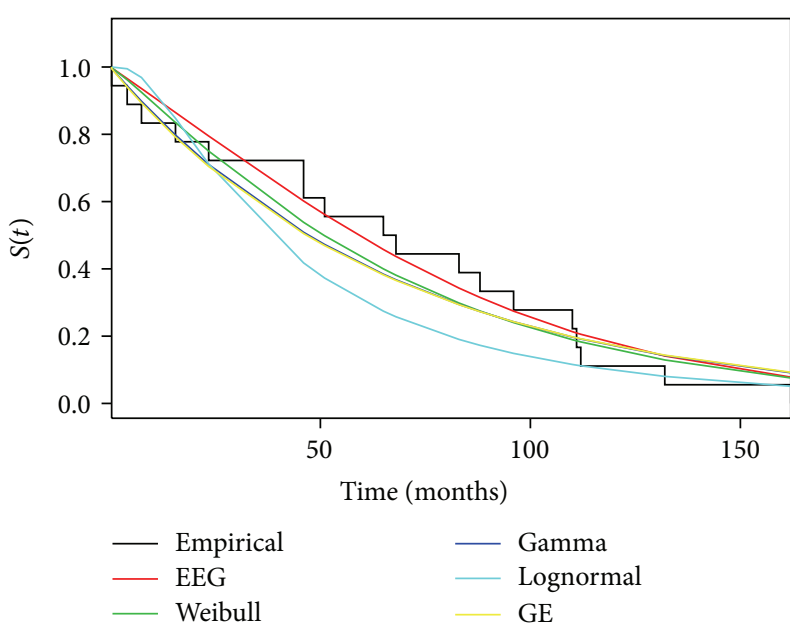

FIGURE 5: Survival function adjusted by different distributions and a nonparametric method considering the data sets related to the ages of 18 patients who died from other causes than cancer.

Considering the MPS estimators, we obtain $\widehat{\lambda}_{\text {MPS }}=$ 0.0065 and $\mathrm{CI}_{95 \%}(\lambda)=(0.0054 ; 0.0077)$ and $\widehat{\gamma}_{\mathrm{MPS}}=14.2279$ and $\mathrm{CI}_{95 \%}(\lambda)=(5.6714 ; 22.7843)$. In Table 5 , we compared the estimates obtained from the different procedures with the MPS estimator in terms of MREs.

From Table 5, we observed that for large samples sizes the estimates are very closer independently of the chosen method. Moreover, due to the large sample size, the MPS estimates and ML estimates are almost the same; such theoretical result is well supported by Cheng and Amin [23]. In Table 6, we have the results from KS test ( $p$ value), AIC, AICc, HQIC, and CAIC, for different probability distributions. Figure 6 presents the survival function adjusted by different distributions and nonparametric survival estimator.

Comparing the empirical survival function with the adjusted distributions, a better fit for the extended exponential geometric distribution among the chosen models

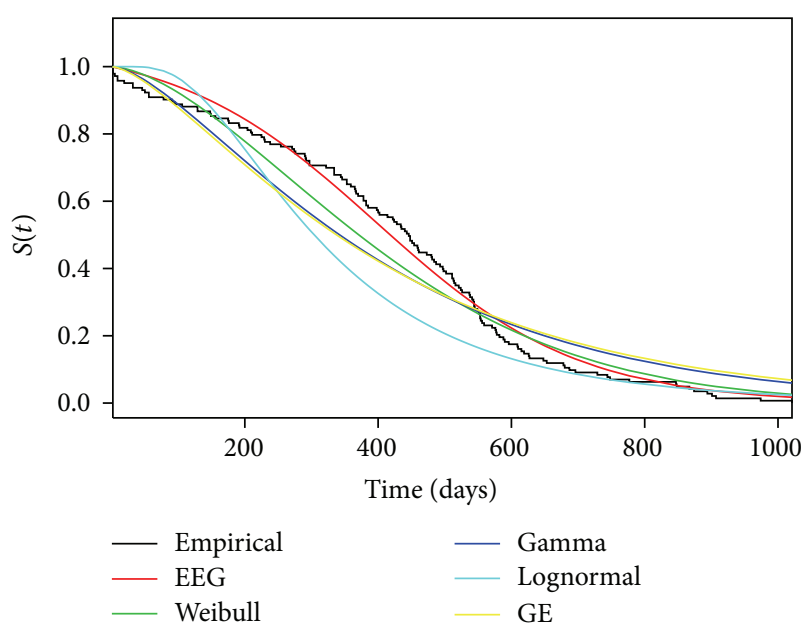

FIGURE 6: Survival function adjusted by different distributions and a nonparametric method considering the data sets related to the serum-reversal time (in days) of 143 children born to HIV-infected mothers.

TABLE 6: Results of the KS test ( $p$ value), AIC, AICc, HQIC, and CAIC for the different probability distributions considering the data set related to the serum-reversal time (in days) of 143 children born to HIV-infected mothers.

\begin{tabular}{lccccc}
\hline Test & EEG & Weibull & Gamma & Lognormal & GE \\
\hline KS & $\mathbf{0 . 6 4 1 3}$ & 0.0067 & 0.0000 & 0.0000 & 0.0000 \\
AIC & $\mathbf{1 9 5 0 . 8 2}$ & 1981.31 & 2001.92 & 2088.90 & 2005.43 \\
AICc & $\mathbf{1 9 5 0 . 9 1}$ & 1981.39 & 2002.01 & 2088.98 & 2005.52 \\
HQIC & $\mathbf{1 9 5 3 . 2 3}$ & 1983.71 & 2004.33 & 2091.31 & 2007.84 \\
CAIC & $\mathbf{1 9 5 8 . 7 5}$ & 1989.23 & 2009.85 & 2096.82 & 2013.36 \\
\hline
\end{tabular}

can be observed. This result is confirmed from AIC, AICc, HQIC, and CAIC, since EEG distribution has the minimum values among the chosen models. Moreover, considering a significance level of 5\%, the EEG distribution was the only 
model in which $p$ values returned from the KS test were greater than 0.05 .

\section{Conclusions}

In this paper, we derived and compared, via intensive simulation study, the estimations of the parameters of the EEG distribution using ten estimation methods. Most importantly, from our simulations, we discovered that the estimates are asymptotically unbiased for the parameters regardless of the estimation method. However, while the ME and CME estimators have, respectively, the largest MREs and MSEs among all the considered estimators, the MPS estimator has the smallest MSEs and the MREs nearest to one, for both parameters, proving to be the most efficient method compared to others for estimating the unknown parameters. As a final advise, combining these results with the good properties of the method such as consistency, asymptotic efficiency, normality, and invariance, we conclude that the MPS estimator is the best one for estimating the parameters of the EEG distribution in comparison with its competitors. Finally, we apply our proposed methodology in two important data sets, demonstrating that the EEG distribution is a simple alternative to be used for lifetime applications.

\section{Competing Interests}

The authors declare that they have no competing interests.

\section{References}

[1] R. D. Gupta and D. Kundu, "Generalized exponential distributions," Australian \& New Zealand Journal of Statistics, vol. 41, no. 2, pp. 173-188, 1999.

[2] K. Adamidis and S. Loukas, "A lifetime distribution with decreasing failure rate," Statistics and Probability Letters, vol. 39, no. 1, pp. 35-42, 1998.

[3] K. Adamidis, T. Dimitrakopoulou, and S. Loukas, "On an extension of the exponential-geometric distribution," Statistics \& Probability Letters, vol. 73, no. 3, pp. 259-269, 2005.

[4] F. Louzada, M. Roman, and V. G. Cancho, "The complementary exponential geometric distribution: model, properties, and a comparison with its counterpart," Computational Statistics \& Data Analysis, vol. 55, no. 8, pp. 2516-2524, 2011.

[5] M. Nassar and N. Nada, "A new generalization of the exponential-geometric distribution," Journal of Statistics: Advances in Theory and Applications, vol. 7, pp. 25-48, 2012.

[6] F. Louzada, V. A. A. Marchi, and M. Roman, "The exponentiated exponential-geometric distribution: a distribution with decreasing, increasing and unimodal failure rate," Statistics, vol. 48, no. 1, pp. 167-181, 2014.

[7] F. Louzada, V. Marchi, and J. Carpenter, "The complementary exponentiated exponential geometric lifetime distribution," Journal of Probability and Statistics, vol. 2013, Article ID 502159, 10 pages, 2013.

[8] H. Bidram, J. Behboodian, and M. Towhidi, "A new generalized exponential geometric distribution," Communications in Statistics-Theory and Methods, vol. 42, no. 3, pp. 528-542, 2013.

[9] P. L. Ramos, F. A. Moala, and J. A. Achcar, "Objective priors for estimation of extended exponential geometric distribution,"
Journal of Modern Applied Statistical Methods, vol. 13, no. 2, pp. 226-243, 2014.

[10] R. D. Gupta and D. Kundu, "Generalized exponential distribution: different method of estimations," Journal of Statistical Computation and Simulation, vol. 69, no. 4, pp. 315-337, 2001.

[11] J. Mazucheli, F. Louzada, and M. E. Ghitany, "Comparison of estimation methods for the parameters of the weighted Lindley distribution," Applied Mathematics and Computation, vol. 220, no. 1, pp. 463-471, 2013.

[12] M. Teimouri, S. M. Hoseini, and S. Nadarajah, "Comparison of estimation methods for the Weibull distribution," Statistics, vol. 47, no. 1, pp. 93-109, 2013.

[13] S. Dey, T. Dey, and D. Kundu, "Two-parameter Rayleigh distribution: different methods of estimation," American Journal of Mathematical and Management Sciences, vol. 33, no. 1, pp. 5574, 2014.

[14] P. Kitidamrongsuk, Discriminating between the Marshall-Olkin exponential distribution and the gamma distribution [Doctor of Philosophy (Statistics)], National Institute of Development Administration, Bangkok, Thailand, 2010.

[15] A. Erdelyi, W. Maguns, F. Oberhettinger, and F. G. Tricomi, Higher Transcendental Functions, McGraw-Hill, New York, NY, USA, 1953.

[16] A. W. Marshall and I. Olkin, "A new method for adding a parameter to a family of distributions with application to the exponential and Weibull families," Biometrika, vol. 84, no. 3, pp. 641-652, 1997.

[17] G. Casella and R. Berger, Statistical Inference, Duxbury, Belmont, Calif, USA, 2nd edition, 2002.

[18] J. H. K. Kao, "Computer methods for estimating weibull parameters in reliability studies," IRE Transactions on Reliability and Quality Control, vol. 13, pp. 15-22, 1958.

[19] J. H. K. Kao, "A graphical estimation of mixed Weibull parameters in life-testing of electron tubes," Technometrics, vol. 1, no. 4, pp. 389-407, 1959.

[20] N. R. Mann, R. E. Schafer, and N. D. Singpurwalla, Methods for Statistical Analysis of Reliability and Life Data, John Wiley \& Sons, New York, NY, USA, 1974.

[21] J. R. Hosking, " $L$-moments: analysis and estimation of distributions using linear combinations of order statistics," Journal of the Royal Statistical Society, Series B-Methodological, vol. 52, no. 1, pp. 105-124, 1990.

[22] R. C. H. Cheng and N. A. K. Amin, "Maximum product of spacings estimation with application to the lognormal distributions," Math Report 79-1, Department of Mathematics, UWIST, Cardiff, UK, 1979.

[23] R. C. H. Cheng and N. A. K. Amin, "Estimating parameters in continuous univariate distributions with a shifted origin," Journal of the Royal Statistical Society, Series B: Methodological, vol. 45, no. 3, pp. 394-403, 1983.

[24] B. Ranneby, "The maximum spacing method: an estimation method related to the maximum likelihood method," Scandinavian Journal of Statistics, vol. 11, no. 2, pp. 93-112, 1984.

[25] R. C. H. Cheng and M. A. Stephens, "A goodness-of-fit test using Moran's statistic with estimated parameters," Biometrika, vol. 76, no. 2, pp. 385-392, 1989.

[26] R. D’Agostino and M. Stephens, Goodness-of-Fit Techniques, Marcel Dekker, New York, NY, USA, 1986.

[27] A. Luceño, "Fitting the generalized Pareto distribution to data using maximum goodness-of-fit estimators," Computational Statistics \& Data Analysis, vol. 51, no. 2, pp. 904-917, 2006. 
[28] P. D. M. Macdonald, "Comment on 'an estimation procedure for mixtures of distributions' by Choi and Bulgren," Journal of the Royal Statistical Society, Series B: Methodological, vol. 33, no. 2, pp. 326-329, 1971.

[29] J. W. Boag, "Maximum likelihood estimates of the proportion of patients cured by cancer therapy," Journal of the Royal Statistical Society, Series B, vol. 11, pp. 15-53, 1949.

[30] A. N. F. Silva, Estudo evolutivo das crianças expostas ao HIV e notificadas pelo Núcleo de Vigilância Epidemiológica do HCFMRP-USP [Dissertação de Mestrado], Faculdade de Medicina de Ribeirão Preto, Universidade de São Paulo, São Paulo, Brazil, 2004. 


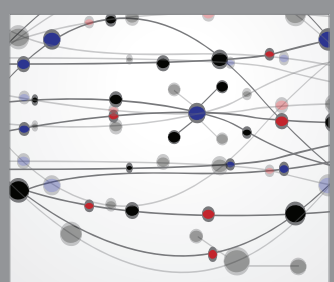

The Scientific World Journal
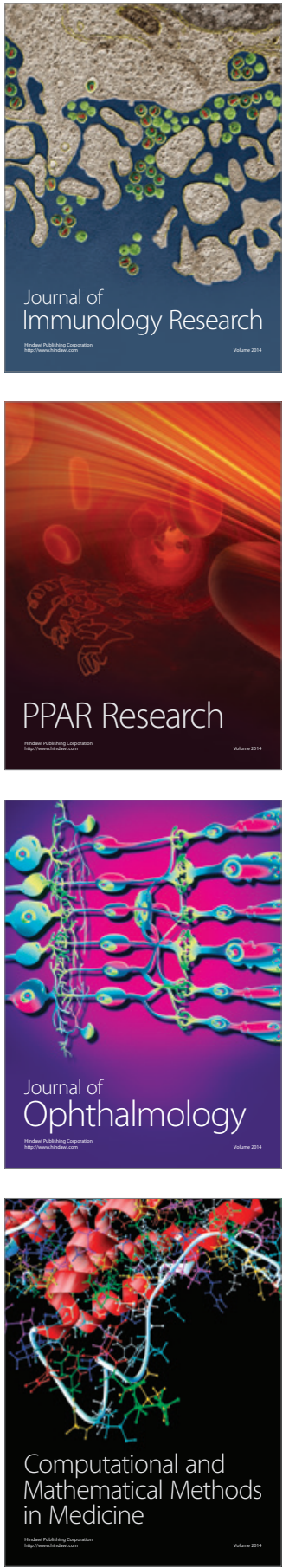

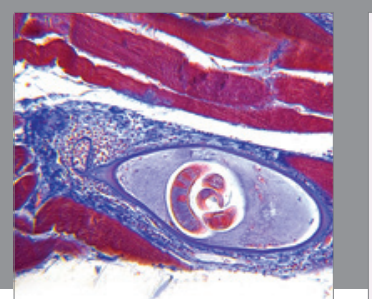

Gastroenterology Research and Practice

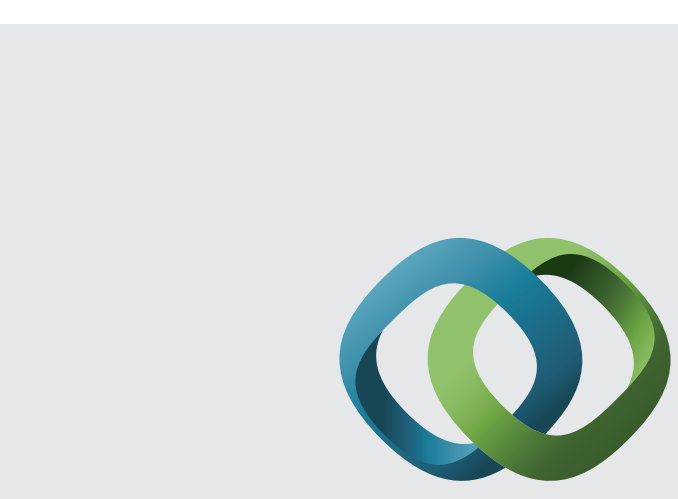

\section{Hindawi}

Submit your manuscripts at

http://www.hindawi.com
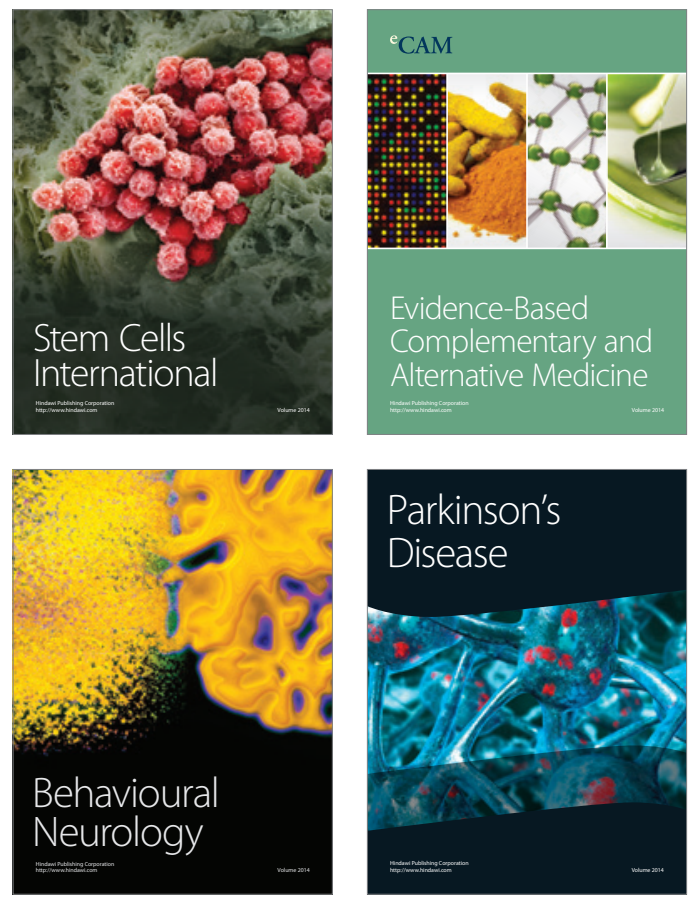
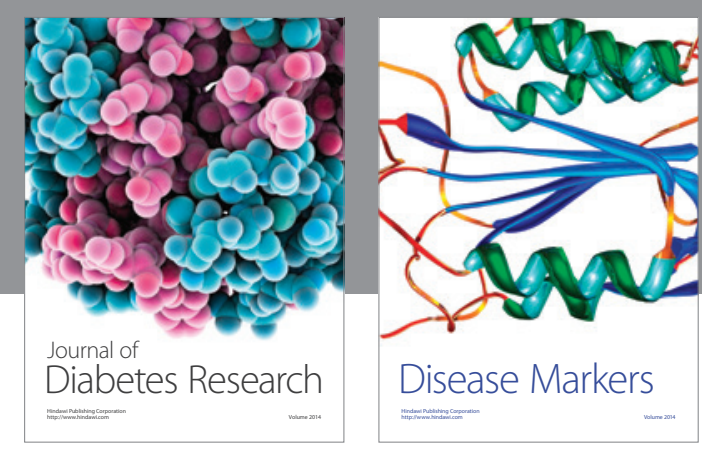

Disease Markers
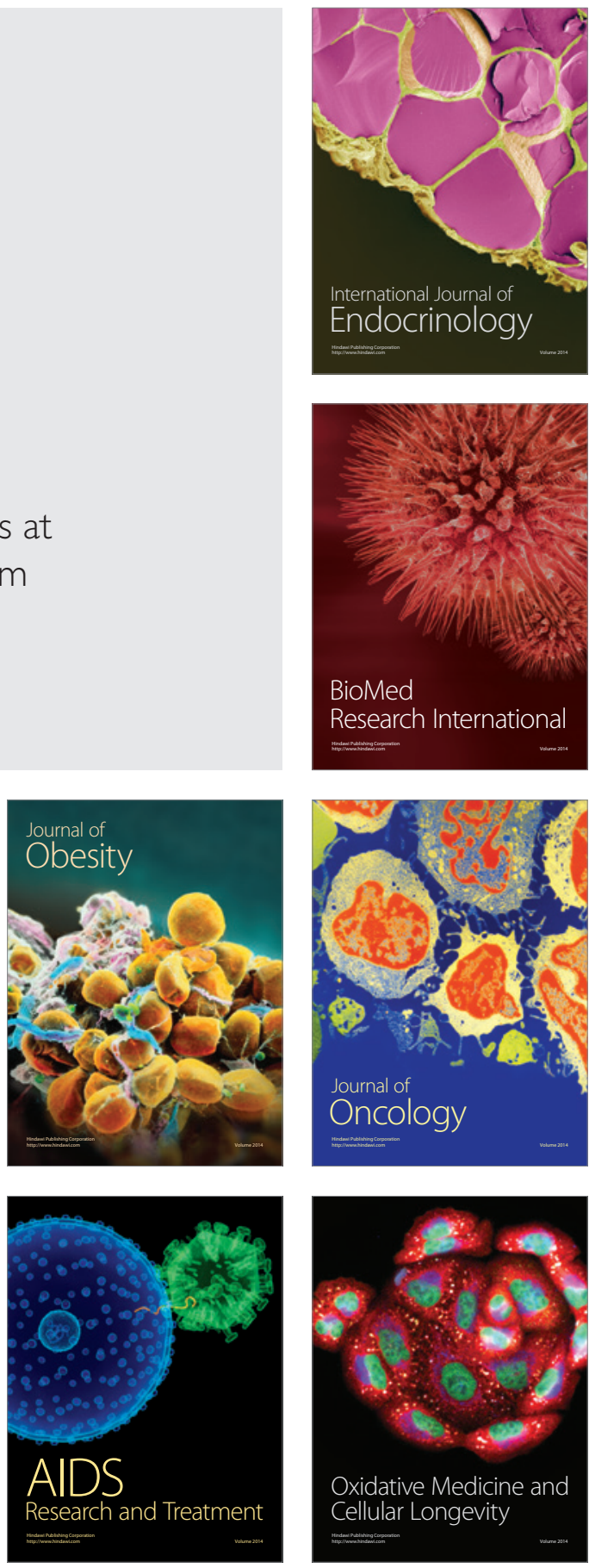